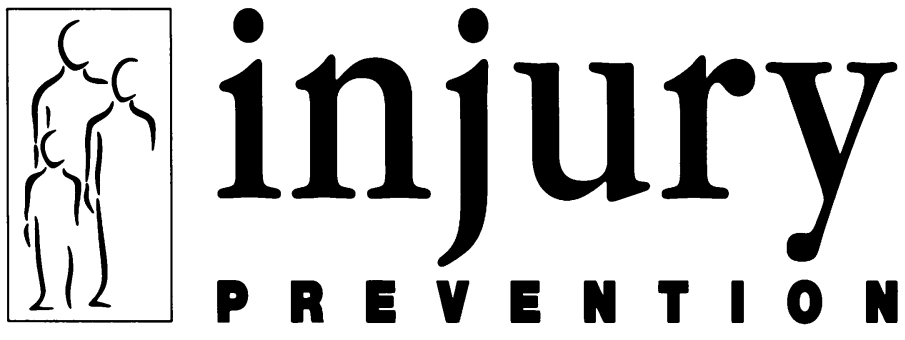

Journal of the International Society for Child and Adolescent Injury Prevention

\title{
Editorials
}

\section{'Interdisciplinary' and 'multidisciplinary' are not synonymous}

One distinctive feature of this journal is our determination to represent the many disciplines involved in injury prevention. Our contributors come from a wide range of professional perspectives. But simply having such diverse representation is no guarantee that their respective strengths will enhance our success in preventing injuries. What is needed is to find a way to ensure that these many skills and viewpoints work together effectively.

Consider, for example, road safety. Engineers continue to make enormous contributions to reduce the chances of crashes by the manner in which motorways are constructed. They place posts on steep curbsides and separate traffic with barriers that have energy absorbent characteristics. Automotive engineers have brought us seat restraints, air bags, and more crash resistant cars. All these have come about because manufacturers have discovered that 'safety sells'. But the list does not end there, of course.

Psychologists help us understand what children can and cannot be expected to learn about road crossing depending on their age and development. Urban planners have invented many traffic calming measures that seem encouraging. However, more rigorous assessments are still needed because too many have used simple before-after designs, open to a range of questions and criticisms. Epidemiologists, along with other researchers, evaluate programmes to identify elements responsible for the most promising results. Equally critical are the contributions of road safety officers, crossing guards, and responsible school bus operators. Although some of these are not professional 'disciplines' in the strict sense of the word, they are all, none the less, players on a winning team.

But why write about a notion most readers already accept as pivotal to prevention? I do so to draw attention to what is missing. Throughout the conference at which the journal was launched (and emerging repeatedly in the rhetoric of most injury prevention zealots, like the recurrence of crescendos in Ravel's Bolero), are the often linked but occasionally misunderstood terms - 'multidisciplinary' and 'interdisciplinary'.

What I have just described are examples of many disciplines working toward the same goal - a multidisciplinary exercise. What I have not described is necessarily interdisciplinary. This means working together, perhaps synergistically, but certainly harmoniously. I believe we will not achieve our ultimate objective as quickly, completely, or efficiently, until we find a way to work in a fully interdisciplinary manner. This is much tougher than it sounds. The reality is that not only does group A often fail to communicate with group B, there are also many examples where $A$ and $B$, while working toward the same goal, are actually in competition - for resources or recognition. In contrast, in a perfectly interdisciplinary world, none of this would ever happen. Groups A, B, to $Z$ would work in harmony, and share in the pride of their mutual accomplishments.

We are a long way from any such situation in most countries. Ragnar Berfenstam's editorial describes how one country has come close to doing so but even the Swedish example falls short of the Utopia I imagine.

When I've had more time to reflect on the matter, and am able to choose my words with great care to ensure that none are misinterpreted I will elaborate on my proposition that the interdisciplinary millennium might best be achieved under a health umbrella. For now, to get you started thinking about this idea, consider the following situation: in many countries the posting of $30 \mathrm{kph}(20 \mathrm{mph})$ speed limits in school zones is left to the discretion of local authorities. Does this make sense? Why should health authorities not have the final say in such matters? All those children (and others, especially the elderly) whose injuries would be prevented by reducing and enforcing these limits, are ultimately a health responsibility. It is the health sector that must treat these cases and on whom the costs of care fall.

One other element of the multi- inter- disciplinary challenge is also reflected in the content of this journal. We want to provide equal prominence for the scientists who write original research reports as for the front line workers whose programmes we feature and whose projects we describe.

Unfortunately, the most difficult challenge for the injury prevention family is to establish a dialogue between these two worlds. This is analogous to CP Snow's 'Two cultures ...'; the town-gown schism; or the split between doers and thinkers. (Although, of course, most doers are thinkers and vice versa.) 
The great paradox of injury prevention is that we already know much about what works but have yet to discover the most effective way to implement what we know. Part of the answer lies in discovering how to make what is now a multidisciplinary process one that is essentially interdisciplinary.

\section{Classics . . . and thanks, again!}

This issue includes the second of a series of injury prevention classics. These 'classics' are copies of papers the editors believe to be important in our field, but which are unlikely to be easily available to most readers because they were published long ago. (At our home an occasional dinner table debate concerns whether something can be properly termed a 'classic' if it is not very old. The semantic purist among us - me - argues that at best, such items should be designated at 'destined to be a classic'.) This aside, our goal for this section is to identify papers that have shaped our ideas, and which more often than not are 'old' by standards of the still young field of injury prevention.

Considering these requirements, it is not surprising but nevertheless sad, that the author of this issue's classic, Stina Sandels, and that which appeared in the previous issue, William Haddon, are both now dead. I hope they would be pleased to know that among their many other important contributions, these papers have now been singled out, and, in a sense, 'immortalized'. Doing so not only helps us as readers, but is also our way of saying thanks to them and the other pioneers who struggled to bring a message to a largely unreceptive audience. We welcome suggestions of similar papers for this honour.

Writing this explanation provides me with an opportunity to deliver a related message. It is that we should all make more of an effort to acknowledge our debts to our older colleagues while they are still alive. We live in a society that eschews reference to death. It is almost as if we fear it will bring bad luck if we break this taboo. But we must struggle to overcome this foolish notion.

At the conference that launched the journal, we payed tribute to Ragnar Berfenstam and Hugh Jackson, and do so again in print. Both are justly recognized as pioneers -
Founding Fathers - and there are Founding Mothers as well - Sandels, is one, and Sue Baker another. This is not just a sentimental suggestion; there is a scientific issue as well. In acknowledging the need for better interdisciplinary relationships as a means to better implementation, we should also recognize the need to maintain strong links with the past. We must not overlook important contributions simply because they are old. Many need to be unearthed, dusted off, re-examined, and sometimes, replicated.

Sandels helped us understand how developmental phenomena are intimately linked with the child's risk of injury in traffic. Why then do some schools still assume 6 year olds can cross roads in a school zone without adult supervision? Jackson showed that child resistant containers prevent poisoning. Why then do many health authorities permit medicines to be dispensed in unsafe containers? Berfernstam demonstrated, as did Haddon, that attention to the environment was a key to effective prevention, especially of pedestrian injuries. Why then do many urban planners continue to give pride of place to cars and ignore the needs of children?

The point is that neither front line workers nor researchers can afford to lose track of our history. There is not enough research money or energy to reinvent the wheel, and although many studies demand replication, others stand alone. It is not just sentiment that should prompt us to thank our elders while they are still able to hear our thanks firsthand. By doing so we help maintain a tradition of excellence in our work and in our research.

IB PLESS Editor 